\title{
Singularity Characteristics of Needle EMG IP Signals
}

\author{
Eric Abel*, Hongying Meng*, Alan Forster** and David Holder*** \\ *Biomedical Engineering Research Group, University of Dundee, Dundee, UK \\ **Walton Centre for Neurology and Neurosurgery, Liverpool, UK \\ *** Department of Clinical Neurophysiology, University College London, UK
}

\begin{abstract}
Clinical electromyography (EMG) interference pattern (IP) signals can reveal more diagnostic information than their constituents, the motor unit action potentials (MUAPs). Singularities and irregular structures typically characterize the mathematically defined content of information in signals. In this paper, a wavelet transform method is used to detect and quantify the singularity characteristics of EMG IP signals using the Lipschitz Exponent (LE) and measures derived from it. The performance of the method is assessed in terms of its ability to discriminate healthy, myopathic and neuropathic subjects and how it compares with traditionally used Turns Analysis (TA) methods and a method recently developed by the authors, Inter-Scale Wavelet Maximum (ISWM). Highly significant intergroup differences were found using the LE method. Most of the singularity measures have a performance similar to that of ISWM and considerably better than that of TA. Some measures such as the ratio of the mean LE value to the number of singular points in the signal have considerably superior performance to both TA methods. These findings add weight to the view that wavelet analysis methods offer an effective way forward in the quantitative analysis of EMG IP signal to assist the clinician in the diagnosis of neuromuscular disorders.
\end{abstract}

\section{Introduction}

The conventional needle electromyographic (EMG) examination is performed using a concentric or monopolar needle electrode. EMG signals can be recorded during varying degrees of voluntary muscle activity, particularly maximum voluntary contraction. With minimal voluntary effort, only a few motor units are activated and the individual motor-unit action potentials (MUAPs) can be identified. As the force of contraction increases, more motor units start to fire and the EMG signal becomes increasingly complex, with the individual MUAPs superimposing upon each other in the signal. Individual MUAPs can no longer readily be identified. This signal is known as an the Interference Pattern (IP).

Analysis of the EMG IP has been shown to be useful in the description of muscle activity, muscle fatigue, occupational work, chronic muscle pain, disused muscle and dystonic muscles treated with botulinum toxin and in the diagnosis of patients with neuromuscular disorders [1]. It is normal clinical practice to examine the IP signal from visual inspection of an 
oscilloscope trace and from listening to the signal through a loudspeaker, as its bandwidth is within the audio frequency range of the human ear. Abnormalities are most easily detected in the more severe cases. In myogenic disorders, due to loss of individual muscle fibres, MUAPs are low in amplitude and short in duration. This manifests as a high-pitched sound from the loudspeaker. In order to compensate for the low amplitude, a large number of motor units are recruited at lower than normal levels of muscle contraction. With increasing force, a full interference pattern is developed at less than maximal contraction [2]. In neurogenic disorders, the excited motor neurons are reduced in number and muscle fibres are reorganised to give larger MUs, which generate MUAPs of higher amplitude and longer duration than normal. In order to maintain a certain force of contraction for this reduced recruitment, the available motor neurons must fire at an appropriately higher than normal rate to compensate for the motor neuron loss. This manifests as a duller sound from the loudspeaker. These perceived frequency changes to the signal in neuromuscular disease conditions may be the reason for some of the earliest quantitative methods being based on frequency domain analysis [3-6].

Time domain methods were introduced subsequently and have been more popular than frequency analysis. Turns Analysis (TA) has for many years been the most commonly used analytical tool for clinical EMG interpretation, both in research and on commercial EMG systems. A turn is defined as a change in EMG signal potential of more than $100 \mu \mathrm{V}$ between adjacent turning points [7]. TA measures include the number of turns (NT) in the IP signal and the mean voltage (amplitude) difference between successive turns (MA). The ratio NT:MA has been found to increase in cases of myopathy and decrease in neuropathies [8].

More recently, a range of elaborate signal processing methods has been developed to try to improve the quantification of the EMP IP signal [9]. These and the many other methods that have been used in IP analysis are reviewed [10]. Very few methods have been of sufficient diagnostic value to be incorporated into commercial EMG instrumentation and none is 
reliable enough to be relied upon without observation of the signal by the clinician. Neural networks have been applied to combine the best of many time and frequency based measures in order to try to improve the diagnostic yield but did not give adequate classification for clinical use [11]. Pathological changes in the signal are often subtle, particularly in the early stages of a disease or in less severe cases, which has made quantification for diagnostic purposes difficult to achieve. Furthermore, the EMG signal is non-stationary. The MUAPs are transients that are not identical for each recurrence of the same MU. For this reason, timefrequency methods are now being used to characterize the localized frequency content of each MUAP [12,13]. These have focused mainly on low force signals, which show the MUAPs rather than the IP. One of the more promising measures for assisting in the clinical diagnosis of neuromuscular disease from analysis of the IP is the Inter-Scale Wavelet Maximum (ISWM) [14]. The method identifies the Wavelet Transform Modulus Maxima, or Wavelet Maxima (WM) [15] associated with each event in the signal, determines which scale is most active and calculates the magnitude of the coefficient at that scale, which is the ISWM. This method has been shown to differentiate myopathic and neuropathic abnormalities. In contrast, more traditional methods used on the same EMG data could not differentiate all the groups.

Singularities and irregular structures typically characterize the mathematically defined content of information in signals. The wavelet transform can identify these local signal structures by virtue of its zooming effect at different scales. The WM can detect all singularities in a signal and contain the necessary information to allow them to be analyzed and characterized [16]. In the EMG IP signal, the rising edge of a MUAP is a result of the synchronous activation of a muscle fibre group, which form the MU. In the multiscale analysis employed in the ISWM method [14] the singularity associated with the rising edge of the MUAP is represented at each scale by one WM. In this paper the Lipschitz Exponent (LE) is used to characterize these singularities by examining their values across scales, in order to determine if abnormal characteristics can be detected in the IP signals from patients with neuromuscular disease. 


\section{Singularity detection and measurement from the wavelet transform}

The LE is the most popular measure of the singularity behavior of a signal. It is a real number that can characterize the local regularity or smoothness in a signal. An local LE value of greater than 1 means that the signal is differentiable at this locality, while a value smaller than 1 means that the signal is not differentiable and has a singularity that is characterized by the value of LE. It is defined and described in detail in [16]. The local signal regularity at regions of singularity can be characterized by the magnitude of the decay of the wavelet maxima amplitudes across scales. Singularities can be detected by following the wavelet Modulus Maxima (WM) lines at fine scales. The LE value of each singularity point, representing rapid change of the signal at that point, gives a measure of the regularity of the signal.

The wavelet transform can detect and measure the both non-oscillating and oscillating singularity. In this paper, only the characteristics of non-oscillating singularities are studied, because singularity points of EMG IP signals do not tend to oscillate. A similar method has been used for edge detection in 2-dimensional image processing, for detecting edges of gray scale images [16]. In the EMG IP signal all the non-oscillating singular points in the signal are identified by the WM lines, from which the LE values are determined. An analysis of these values and their statistical distribution in a signal could provide a meaningful quantitative characterization of singularity in the EMG IP for identifying neuromuscular disease abnormalities.

The uniform Lipschitz regularity of a signal on an interval can be related to the amplitude of its WM values at fine scales $[15,16]$. The wavelet transform $W f(u, s)$ of signal $f(t)$ may be expressed as: 


$$
W f(u, s)=\int_{-\infty}^{+\infty} f(t) \frac{1}{\sqrt{s}} \psi^{*}\left(\frac{t-u}{s}\right) d t
$$

where $\psi(t)$ is a mother wavelet function, $\psi^{*}(t)$ is its conjugate, $s>0$ is the scale factor and $u$ is the spatial position. Then, from [15], if a function $f \in L^{2}(R)$ is uniformly Lipschitz $\alpha \leq n$ (where $n$ is an integer) over the interval $[a, b]$, where a and $\mathrm{b}$ are real values, there exists a value of $\mathrm{A},(A>0)$ such that

$$
\forall(u, s) \in[a, b] \times R^{+}, \quad|W f(u, s)| \leq A s^{\alpha+\frac{1}{2}},
$$

where $\mathrm{R}^{+}$is a positive real number. Conversely, if $W f(u, s)$ satisfies equation (2) and if $\alpha<n$ is not an integer then $f$ is uniformly Lipschitz $\alpha$ on $[a+\varepsilon, b-\varepsilon]$ for any $\varepsilon>0$. This implies that $f(t)$ is uniformly LE $\alpha$ in the neighbourhood of $v$ if and only if there exists $A>0$ such that each modulus maximum $(u, s)$ in the region $|u-v| \leq C s$ satisfies the condition

$$
|W f(u, s)| \leq A s^{\alpha+\frac{1}{2}}
$$

where $\mathrm{C}$ is a constant. This may be expressed in logarithmic form as

$$
\log _{2}|W f(u, s)| \leq \log _{2} A+\left(\alpha+\frac{1}{2}\right) \log _{2} s
$$

The Lipschitz regularity $\left(\alpha+\frac{1}{2}\right)$ at $v$ is thus the maximum slope of $\log _{2}|W f(u, s)|$ as a function of $\log _{2} s$ along the WM lines that converge to $v$. In practice, the finest scale ( $\left.s=1\right)$ of the wavelet transform is limited by the resolution of the discrete data, so LE $\alpha$ is estimated from the wavelet transform $W f(u, s)$ at scales $s>1$ to give an estimate of the singularity 
exponent at $\mathrm{s}=1$. The exponent $\alpha+\frac{1}{2}$ is calculated by measuring the decay slope of $\log _{2}|W f(u, s)|$ as a function of $\log _{2} s$ at the finest scale.

A coarse-to-fine approach has been used to create the Wavelet Maxima (WM) line structures. For each WM at the coarsest scale, the WM lines are constructed from the coarser to the finer scales by finding the corresponding WM nearby. A threshold value $\beta$ at the coarsest scale is chosen whereby the absolute value of a local maximum point must be greater than $\beta$ percent of the absolute value of the whole wavelet coefficient in that scale. In the $15 \mathrm{~ms}$ example signal shown in Fig. 1, there are $4 \mathrm{WM}$ points found at scale $2^{5}$ using a threshold $\beta=80 \%$ and WM lines were constructed from scale $2^{5}$ to scale $2^{1}$. These 4 WM lines were associated with 4 singularity points in the signal.

The implementation algorithm for obtaining the LE of the IP signals involves four steps:

(i) Decomposition of signal into different scales by the continuous wavelet transform:

EMG IP waveforms are decomposed to wavelet coefficients by the continuous wavelet transform of the B-spline wavelet. The wavelet transform used here $\left(s=2^{j}, u=k\right)$ is based on the filters of Unser and Aldroubi [17,18]. The first derivative of the cubic B-spline is suited to the detection of gradient changes. This analysis is restricted to dyadic scales $\left(s=2^{j}\right)$ as they offer an efficient way to examine a wide range of frequencies with wavelet transforms. This implementation is a fast algorithm in which continuous wavelet transform is carried out by a discrete scheme [19].

(ii) Detection of the WM points in the characteristic scales: 
The scales selected were $2^{1}, 2^{2}, 2^{3}$ and $2^{4}$, based on a sampling rate for the signals of $32 \mathrm{kHz}$. These finer scales contain the important information for computing the singularity exponent and for obtaining the best singularity estimation. At each characteristic scale, the wavelet maximum points are detected. A threshold $\beta$ was applied, whereby the largest $\beta \%$ of wavelet coefficients at the coarsest scale were selected. The larger the threshold, the fewer the number of local maxima points, but those that remain represent the faster changes in the waveform.

(iii) Determination of WM lines:

The LE values are estimated from the scales $\left[2^{2}, 2^{3}\right]$. The scales $\left[2^{1}, 2^{2}\right]$ were not chosen because of noise at scale $2^{1}$. Initial experimentation using scales $2^{3}, 2^{4}$ and $2^{5}$ as the coarsest scale were found to give very similar results so only results with coarsest scales $2^{4}$ and $2^{3}$ are reported here.

(iv) Calculation of the singularity exponent for each WM point along the WM lines:

The first order derivative of a fourth-order B-spline was selected. This wavelet only has one vanishing moment. Each LE value is found from the WMs along a WM line from scales $s \in\left[2^{2}, 2^{3}\right]$. With the first scale excluded due to the influence of noise, the lowest scale was chosen as $2^{2}$. From equation (4), the LE value can be estimated by the following:

$$
\alpha \approx \log _{2}\left|W f\left(u_{2^{3}}^{v}, 2^{3}\right)\right|-\log _{2}\left|W f\left(u_{2^{2}}^{v}, 2^{2}\right)\right|-\frac{1}{2}
$$

in which $u_{{ }_{2}^{j}}^{v}$ is the WM point in the scale $2^{j}$ associated with the time point $v$ of the IP signal. 
Figure 2 shows a $30 \mathrm{~ms}$ segment of an EMG IP signal and the LE values for each of the six singularity points in this segment, as identified by the wavelet transform. The signal clearly has rapidly changing characteristics at these points. The smaller the LE value, the faster the waveform change. For example, in this picture, the third point has a LE value 0.2907, representing the most rapidly changing singularity. For all the other parts of the segment, the signal has smaller changes with LE values that can be regarded as 1 . From this figure, it can also be seen that this not influenced by any high frequency signal components, which could be associated with noise, as these are not detected as singularity points.

\section{Singularity measures}

Several statistical parameters based on singularity measures were investigated. These were: the Number of Singularity Points (NSP) in the signal, which in some sense describes the level of complexity of the signal; the mean value (Mean) of all the LE values and the standard deviation (SD); the SD/Mean because of its normalizing property; and the ratio of Mean to NSP (Mean/NSP). The latter measure was chosen because it was anticipated that, for myopathy subjects, Mean would be small and NSP large, while the converse should be true for neuropathy subjects.

An additional measure was used to describe the information obtained from the distribution of LE values. In any EMG IP signal, there are hundreds of singularity points with their own LE values, which are in the interval from zero to one. Fig. 3 gives an example for an EMG IP signal of 4 seconds duration. The distribution of LE values was obtained by dividing the inerval $[0,1]$ into 50 segments. Fig. 4 shows an example, using the data from the signal in Fig 3. 
A Principal Component Analysis (PCA) method was used to analyze this distribution vector, which has a dimension of 50. The extracted measure, named the First Principle Component Analysis (FPCA), was the first component of the PCA transformation of the distribution vector. The PCA or Karhunen-Loeve expansion is the best known linear feature extractor that computes several largest eigenvectors of the covariance matrix of the original patterns, i.e. for the original patterns being centred $\left(\sum_{j=0}^{N} x_{j}=0\right)$, PCA diagonalizes its covariance matrix

$$
C=\frac{1}{N} \sum_{j=1}^{N} x_{j} x_{j}^{T}
$$

\section{Experimental Results}

Interference pattern data from concentric needle electrodes were sampled at $32 \mathrm{kHz}$ from the analogue output of a Medelec MS60 EMG recording system (Oxford Instruments, Oxford, UK). Four seconds of data from five sites were examined from the biceps muscles of 11 healthy subjects, 26 patients with myopathic disease and 20 patients with neuropathic disease, at maximum voluntary muscle contraction. The recording sites were obtained in the usual clinical manner, by removing the needle from the muscle, without withdrawing it completely through the skin, then reinserting the needle into the same muscle. The Medelec MS60 has a built-in low pass filter with a cut-off frequency of $10 \mathrm{kHz}$ (which functioned as an antialiasing filter) and a high pass filter with a cut-off frequency of $30 \mathrm{~Hz}$ for removal of low frequency noise artifacts. Patient diagnoses were based on a range of clinical information, which included a general examination and clinical history of the patient, and EMG and nerve conduction tests. Muscle biopsies were not taken in the majority of cases, on ethical grounds, as they are only considered in our EMG Clinic in cases where diagnosis is uncertain or for specific clinical reasons. 
Data from the five sites of each subject were used to generate descriptive statistical measures to measure the distribution of the magnitudes of the LE values. The nonparametric MannWhitney U-test [20] was used to test the significant differences between samples from two independent groups for the above measures.

\section{(i) LE Method}

The LE values for all WM lines in a signal were obtained and the mean and standard deviation used to provide basic measures of their distribution of values. This is where we need to show example frequency distributions for each group, in a new figure. Tables 1 to 3 show p-values to three decimal places using examples of different coarsest scales and threshold values. Significant differences $(\mathrm{p}<0.01)$ between Neuropathies $(\mathrm{Ne})$ and the other two groups, Normals (No) and Myopathies (My), were found for many of the measures. For most measures, myopathy subjects show significant differentiation from the other two groups. NSP shows significant difference for neuropathy subjects compared to those from other two groups. Mean/NSP showed the best performance in differentiating all three groups. A comparison of the results in Tables 1 and 2, for scales 4 and 3 respectively as the coarsest scale for determining LE, and of the results in Tables 2 and 3 for thresholds of $70 \%$ and $80 \%$ shows only small differences in performance for most measures. The most difficult groups to differentiate were normal from neuropathy. Using a coarsest scale of $2^{4}$ and a threshold of $70 \%$ (Table 1) gave the best differentiation, with two measures (NSP and Mean/NSP) that could differentiate at $\mathrm{p}=0.002$ or better. 
Table 1: p-values for statistical differences in measures between subject groups with the coarsest scale $2^{4}$ and $\beta=70 \%$.

\begin{tabular}{|l|c|c|c|c|c|c|}
\hline Measure & \multicolumn{2}{|c|}{ No-My } & \multicolumn{2}{c|}{ No-Ne } & \multicolumn{2}{c|}{ My-Ne } \\
\hline & mean & $p$ & mean & $p$ & mean & $p$ \\
\hline Mean & & 0.001 & & 0.710 & & 0.000 \\
\hline SD & & 0.006 & & 0.216 & & 0.033 \\
\hline SD/Mean & & 0.003 & & 0.283 & & 0.007 \\
\hline NSP & & 0.690 & & 0.001 & & 0.000 \\
\hline Mean/NSP & & 0.001 & & 0.002 & & 0.000 \\
\hline FPCA & & 0.052 & & 0.079 & & 0.000 \\
\hline
\end{tabular}

Table 2: p-values for statistical differences in measures between subject groups with the coarsest scale $2^{3}$ and $\beta=$ $70 \%$.

\begin{tabular}{|l|c|c|c|c|c|c|}
\hline Measure & \multicolumn{2}{|c|}{ No-My } & \multicolumn{2}{c|}{ No-Ne } & \multicolumn{2}{c|}{ My-Ne } \\
\hline & mean & $p$ & mean & $p$ & mean & $p$ \\
\hline Mean & & 0.001 & & 0.536 & & 0.001 \\
\hline SD & & 0.022 & & 0.248 & & 0.097 \\
\hline SD/Mean & & 0.009 & & 0.248 & & 0.018 \\
\hline NSP & & 0.288 & & 0.006 & & 0.000 \\
\hline Mean/NSP & & 0.001 & & 0.039 & & 0.000 \\
\hline FPCA & & 0.001 & & 0.665 & & 0.000 \\
\hline
\end{tabular}

Table 3: p-values for statistical differences in measures between subject groups with the coarsest scale $2^{3}$ and $\beta=80 \%$.

\begin{tabular}{|l|c|c|c|c|c|c|}
\hline Measure & \multicolumn{2}{|c|}{ No-My } & \multicolumn{2}{c|}{ No-Ne } & \multicolumn{2}{c|}{ My-Ne } \\
\hline & mean & $p$ & mean & $p$ & mean & $p$ \\
\hline Mean & & 0.001 & & 0.773 & & 0.000 \\
\hline SD & & 0.017 & & 0.201 & & 0.207 \\
\hline SD/Mean & & 0.007 & & 0.216 & & 0.034 \\
\hline NSP & & 0.335 & & 0.006 & & 0.000 \\
\hline Mean/NSP & & 0.001 & & 0.029 & & 0.000 \\
\hline FPCA & & 0.002 & & 0.788 & & 0.000 \\
\hline
\end{tabular}

(ii) Turns Amplitude Method 
For comparison, more traditionally used measures for EMG analysis were applied to the data.

Turns analysis is a standard method for EMG IP quantification, which has been used for over 30 years and is regarded as a reference against which to compare new methods. A basic turns amplitude analysis $[7,21]$ was performed using the mean amplitude (MA), the Number of Turns (NT) and the ratio of the two (MA/NT). The results are presented in Table 4. The MA/NT measure could not differentiate any of the groups, while the MA could only differentiate the neuropathies from the myopathies and NT could only differentiate the myopathies from the normals.

Table 4: p-values for statistical differences in traditional measures MA, NT and MA/NT between subject groups.

\begin{tabular}{|l|c|c|c|c|c|c|}
\hline Measure & \multicolumn{2}{|c|}{ No-My } & \multicolumn{2}{c|}{ No-Ne } & \multicolumn{2}{c|}{ My-Ne } \\
\hline & mean & $p$ & mean & $p$ & mean & $p$ \\
\hline MA & & 0.027 & & 0.353 & & 0.023 \\
\hline NT & & 0.280 & & 0.577 & & 0.499 \\
\hline MA/NT & & 0.148 & & 0.386 & & 0.388 \\
\hline
\end{tabular}

The results in Tables 1-3 show considerably better performance than those from Turns Analysis.

(iii) ISWM Method

An outline of the ISWM method is described briefly here. A full description of the method can be found in [14]. It uses a fine-to-coarse scale processing of the WM, incorporating a weighting function to associate each WM at a fine scale with only one WM at the next coarser scale The weighting function takes into account the relative magnitudes and temporal proximity of all WM at the coarser scale in this association process, to determine which WM, if more than one is present, should propagate at the next scale. This results in a meaningful propagation of the WMs across scales and enables the construction of a tree structure. The weighting function is a cubic B-spline, which is similar to a Gaussian function [17] but much easier to manipulate computationally. The root of the WM tree is the scale beyond which no corresponding WM can be found. 
Each WM tree consists of one or more WM at each of the $j$ scales. If $N_{j}$ is the number of WM coefficients that belong to a particular WM tree at scale $2^{j}$ and $x_{\lambda}$ are the positions, this group $G_{j}$ is defined by:

$$
G_{j}=\left\{m_{\lambda}=\mid W f\left(x_{\lambda}, 2^{j}\right), \lambda=1 \ldots N_{j}\right\}
$$

The $\lambda$ wavelet coefficients are summed, i.e. $\sum m_{\lambda}, \lambda=1 \ldots N_{j}$. A similar summation is done for all the other scales and the ISWM value is the summation that has the highest value:

$$
I S W M=\max \sum_{j} m_{\lambda}
$$

Fig. 5 shows a 15 ms segment of an EMG IP in the region of an MUAP, the derived WM and the ISWM, calculated from equation (6).. The signal has been decomposed at five scales. The ISWM method uses a fine-to-coarse algorithm to construct a tree structure, as opposed to the coarse-to-fine algorithm used for the singularity method in this paper, which constructs a WM line. The MUAP corresponds to one WM at the coarsest dyadic scale, while at finer scales more than one WM indicates a MUAP with a more complex shape, which is commonly the case in neuromuscular disorders, where there tend to me more rising edges in a MUAP and therefore more WM, with a tendency also for the WM tree to branch out more than for normal EMG signals. At each scale the WM are summed, the highest resulting value being the ISWM. In the example, the ISWM is at scale $2^{4}$.

Table 5: p-values for statistical differences in ISWM measures in scales $2^{2}, 2^{3}$ and $2^{4}$ between subject groups, using scale dependent thresholding

\begin{tabular}{|l|c|c|c|c|c|c|c|c|c|}
\hline Measure & \multicolumn{3}{|c|}{ No-My } & \multicolumn{3}{|c|}{ No-Ne } & \multicolumn{3}{|c|}{ My-Ne } \\
\hline Scale & $\mathbf{2}^{\mathbf{3}}$ & $\mathbf{2}^{\mathbf{4}}$ & $\mathbf{2}^{\mathbf{5}}$ & $\mathbf{2}^{\mathbf{3}}$ & $\mathbf{2}^{\mathbf{4}}$ & $\mathbf{2}^{\mathbf{5}}$ & $\mathbf{2}^{\mathbf{3}}$ & $\mathbf{2}^{\mathbf{4}}$ & $\mathbf{2}^{\mathbf{5}}$ \\
\hline Mean & 0.006 & 0.013 & 0.005 & 0.087 & 0.027 & 0.292 & 0.000 & 0.000 & 0.000 \\
\hline SD & 0.061 & 0.075 & 0.052 & 0.050 & 0.005 & 0.037 & 0.000 & 0.000 & 0.000 \\
\hline Skewness & 0.252 & 0.158 & 0.584 & 0.003 & 0.001 & 0.132 & 0.001 & 0.004 & 0.303 \\
\hline SD/Mean & 0.158 & 0.213 & 0.115 & 0.353 & 0.820 & 0.180 & 0.019 & 0.375 & 0.938 \\
\hline
\end{tabular}

In the ISWM method, noise reduction thresholding is applied at all scales. Only WM with amplitudes greater than the $100 \alpha$-th percentile threshold at each scale are selected for determining the tree structure, where $\alpha$ varies between zero and unity and can be constant across all scales or have a scale-dependent value. Scale dependent thresholding was found to give better performance than a constant $\alpha$ at all scales. The p-values shown in Table 5, comparing the subject groups for statistical measures obtained from an analysis of the ISWM 
values, show the best results obtained from the earlier study [14] (with $\alpha_{1}=0.95, \alpha_{2}=0.85$, $\left.\alpha_{3}=0.75, \alpha_{4}=0.65, \alpha_{5}=0.55\right)$. As with the LE results, there is good discrimination between the normals and the neuropathies, and between the myopathies and the neuropathies, even at $\mathrm{p}<0.01$.

The ISWM by definition occurs at one scale only, information at other scales being discarded. The LE measure is calculated from two adjoining scales so provides complementary, between-scale, information about the EMG IP signals.

In order to test the more general effectiveness of the LE method, a larger dataset was analysed, using a wider range of muscle groups. EMG IP signals were recorded from 271 patients. The muscle sites includes tibialis anterior, EDC, extensor proprius indicis, APB, ECR, triceps, pronator teres, flexor pollicis longus, pronator teres, lateral gastrocnemius, soleus, adductor magnus, the short head of biceps femoris, the long head of biceps femoris, lateral peroneus, iliopsoas and biceps brachii. The results are shown in Table 6. These results showed a consistently good performance and in particular give improved confidence over FPCA. in discriminating patients with neuropathies from normal subjects.

Table $6 \mathrm{p}$-values for statistical differences in measures between subject groups with the coarsest scale $2^{3}$ and $\beta=70 \%$.

\begin{tabular}{|l|c|c|c|c|c|c|}
\hline Measure & \multicolumn{2}{|c|}{ No-My } & \multicolumn{2}{c|}{ No-Ne } & \multicolumn{2}{c|}{ My-Ne } \\
\hline & mean & $p$ & mean & $p$ & mean & $p$ \\
\hline Mean & & 0.000 & & 0.607 & & 0.000 \\
\hline SD & & 0.000 & & 0.879 & & 0.000 \\
\hline SD/Mean & & 0.000 & & 0.851 & & 0.000 \\
\hline NSP & & 0.001 & & 0.000 & & 0.000 \\
\hline Mean/NSP & & 0.000 & & 0.000 & & 0.000 \\
\hline FPCA & & 0.000 & & 0.189 & & 0.000 \\
\hline
\end{tabular}

5. Discussion and Conclusion 
In this paper, a wavelet transform method has been used to detect and quantify the singularity characteristics of EMG IP signals and some new quantitative measures have been derived. The LE measure uses a coarse to fine algorithm, which is faster computationally than a fine to coarse algorithm, which is implemented in the ISWM method. Furthermore, only the WM points at the coarsest scales need to be kept to construct the WM lines, whereas the fine to coarse algorithm requires all WM points to be analysed in order to construct a WM tree structure. Both algorithms perform considerably better than the TA method in differentiating the subject groups and they may complement each other in a future neural network or other artificial intelligence system that uses multiple measures for diagnostic purposes.

The Mean/NSP derived from the LE performed particularly well. The following explanation may be put forward for the fact that the Mean/NSP showed the best performance. In neuropathies there are generally fewer but larger MUAPs than in normal subjects so NSP is smaller and Mean is higher (because the waveform is smoother). In myopathies there are more MUAPS, but these are smaller in magnitude, so the NSP is larger and the Mean is smaller (because the waveform is not smooth). The ratio of Mean/NSP could therefore be expected to discriminate the groups, which was found to be the case.

From wavelet transform theory, it is unrealistic to try to estimate every singularity exactly and individually from a signal. However, the singularity of most singularity points in the EMG IP can be detected and estimated by this method. The wavelet transform can detect and measure both non-oscillating and oscillating singularity. In this paper, only the former has been studied because we aimed to obtain the global singularity property of the EMG signals, which is best represented by the non-oscillating component. However, in order to obtain the complete singularity characteristics of the signal, the oscillating singularity should also be studied. It might provide more detailed information about local signal abnormality which could assist in disease detection. As the method stands, highly significant differences in the non-oscillating singularity measures have been found between healthy, myopathic and neuropathic subjects. 
These findings make a contribution to enhancing the diagnostic capability of EMG IP signal analysis, which remains a difficult process. 


\section{List of Figures}

Fig.1. Coarse to fine method to find WM lines for a $15 \mathrm{~ms}$ EMG IP signal. IP signals were decomposed into 5 scales and $4 \mathrm{WM}$ lines were constructed from coarser to finer scales. Each WM line represents a singularity in the signal

Fig.2. A 30 ms EMG IP signal with 6 singularity points marked as circles and LE value for every singularity

Fig.3 LE values in an EMG IP signal. There are 1775 values, each in the interval $(0,1)$. The smaller the LE value, the faster the waveform change at this point.

Fig.4 Distribution of LE values of EMG IP signals. The interval $(0,1)$ were divided into 50 sub-intervals and the number of singularity points within each sub-intervals were calculated.

Fig 5. A 15 ms signal segment containing a MUAP with its WM tree. At each scale, the summation of WM is computed. The largest value is the ISWM. 


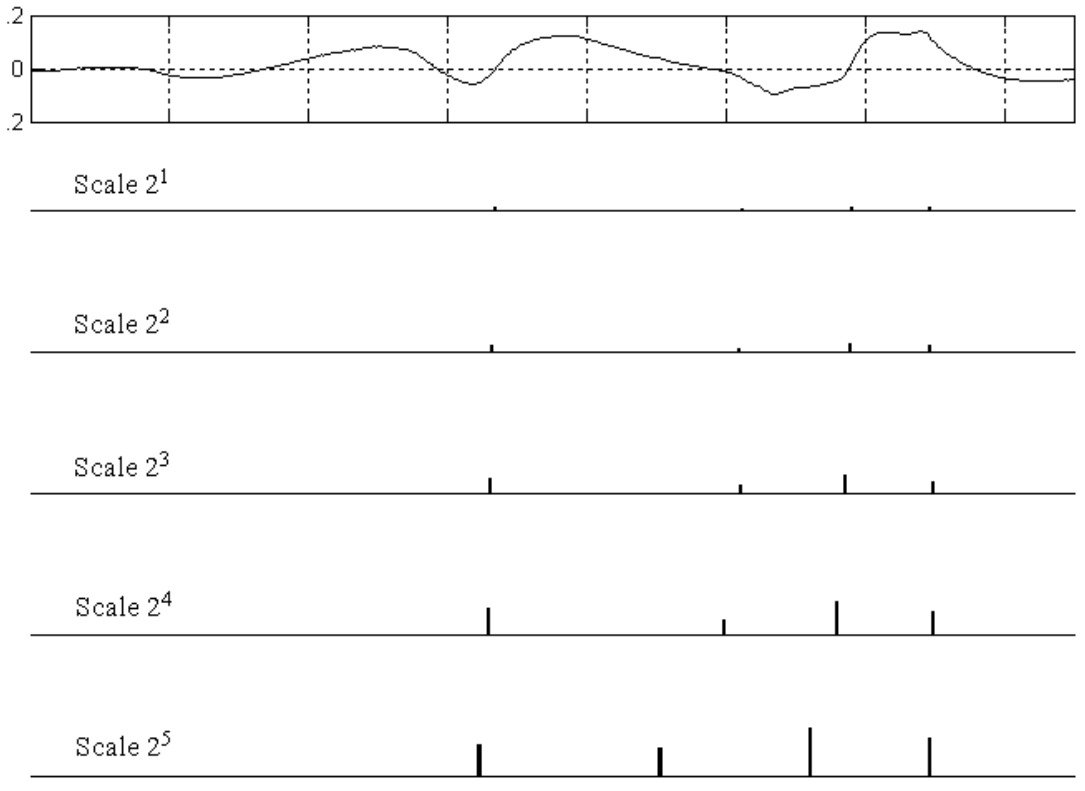

Fig.1. 


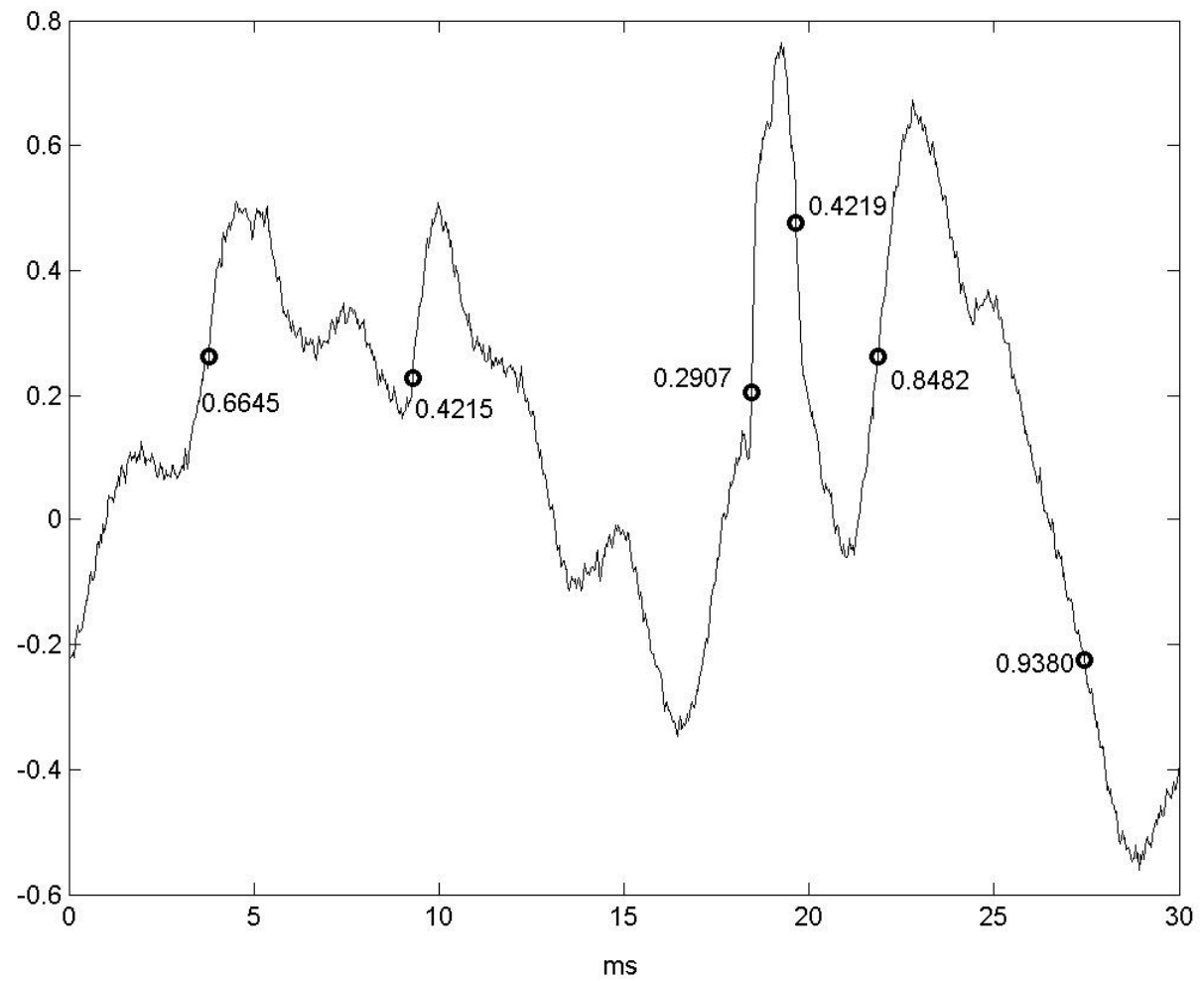

Fig 2 


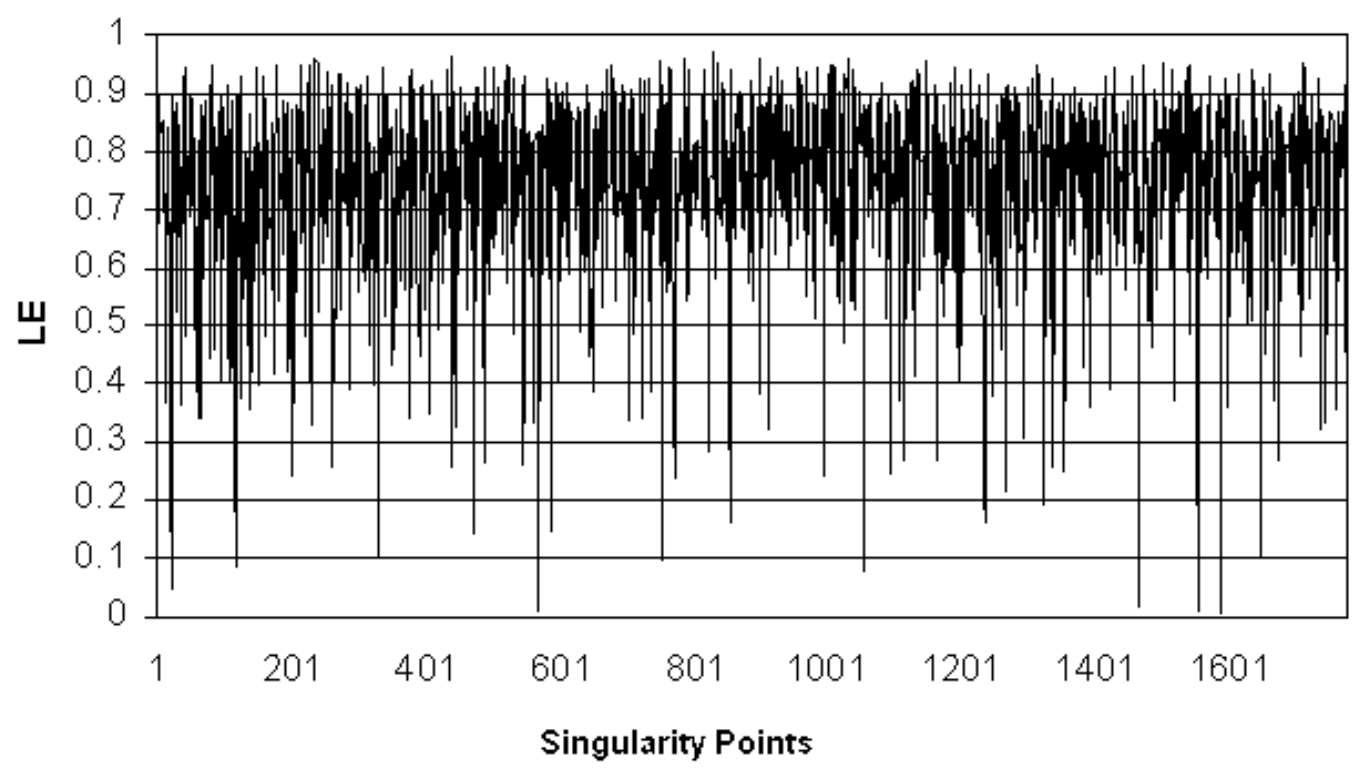

Fig. 3 


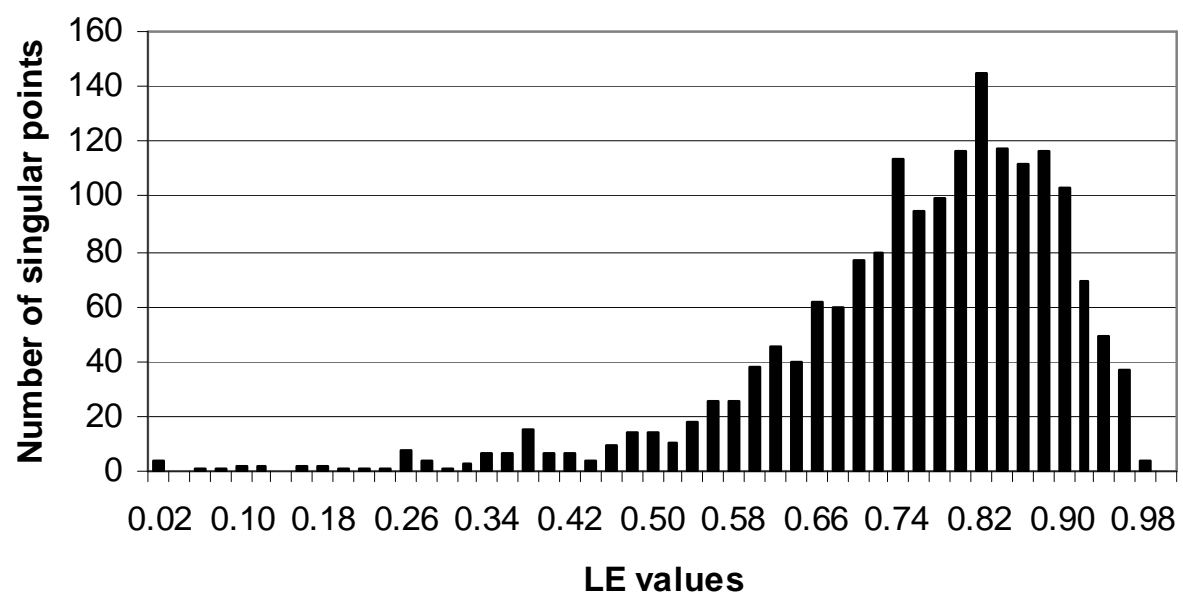

Fig.4 


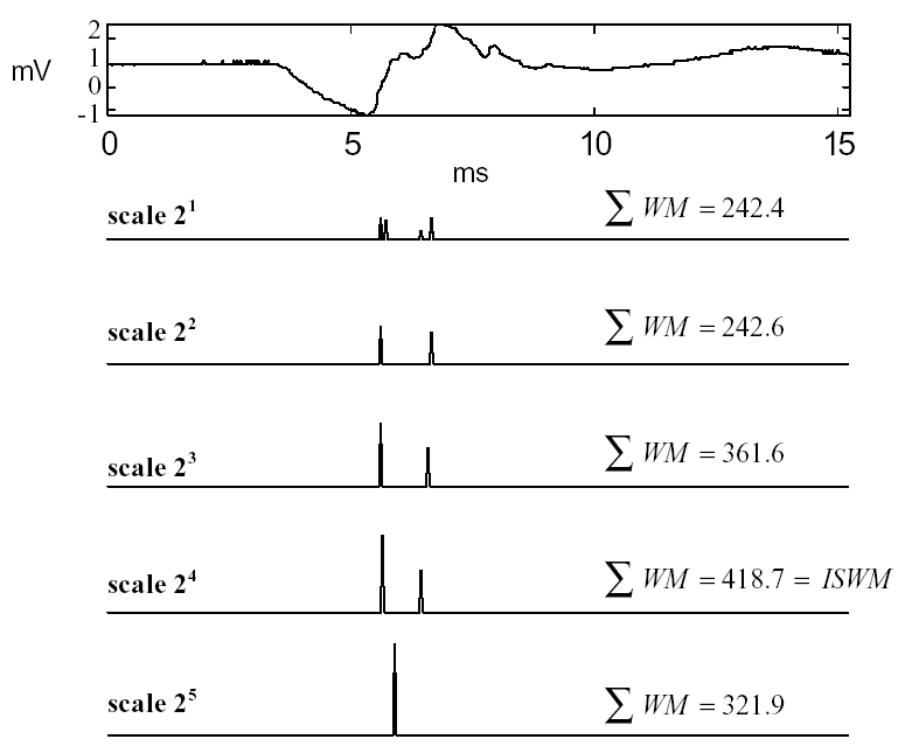

Fig 5. 


\section{References}

[1] A. Fuglsang-frederiksen, the utility of interference pattern analysis, Muscle and Nerve, January, 2000, pp:18-36.

[2] J. Kimura, "Electrodiagnosis in Diseases of nerve and muscle: Principle and practice", Davis F. A., 1983.

[3] A. Fuglsang-Frederiksen, "Power Spectrum of the Needle EMG in Normal and Diseased Muscles", Methods in Clinical Neurophysiology, vol. 2, pp. 1-8, 1990.

[4] H. Christensen and A. Fuglsang-Frederiksen, "Power spectrum and turns analysis of the EMG at different voluntary efforts in normal subjects", Electroencephalogr. Clin. Neurophysiol., vol. 64, pp. 528-535, 1986.

[5] J. Fex, and C. E.T. Krakau, "Some experiences with Walton's frequency analysis of the electromyogram”, J. Neurol. Neurosurg. Psychiat., vol. 20, pp. 178-184, 1957.

[6] J. N. Walton, "The electromyogram in myopathy: analysis with the audio-frequency spectrometer", J. Neurol. Neurosurg. Psychiat., vol. 15, pp. 219-226, 1952.

[7] L. E. Larsson, "On the relation between the EMG frequency spectrum and the duration of symptoms in lessions of the peripheral motor neuron", Electroencephalogr. Clin. Neurophysiol., vol. 38, pp. 69-78, 1975.

[8] A. Fuglsang-Frederiksen, U. Scheel and F. Buchthal, "Diagnostic yield of the analysis of the pattern of electrical activity of muscle and of individual motor unit potentials in neurogenic involvement", J. Neurol. Neurosurg. Psychiatry, vol. 40, pp. 544-554, 1977.

[9] D. B. Sanders, E. V. Stalberg and S. D. Nadedkar, Analysis of the electromyographic interference pattern, Journal of clinical neurophysiology, 13(5): 385-400,1996.

[10] J. Finsterer, "EMG-interference pattern analysis", Journal of Electromyography and Kinesiology, vol 11, pp 231-246. 2001

[11] E. W. Abel, P. C. Zacharia, A. Forster and T. L. Farrow, "Neural network analysis of the EMG interference pattern", Med. Eng. Phys., vol. 18, issue 1, pp. 12-17, 1996.

[12] C. S. Pattichis and M. S. Pattichis, "Time-Scale Analysis of Motor Unit Action Potentials", IEEE Trans. Biomed. Engineer., vol. 46, no. 11, pp. 1320-1329, Nov. 1999.

[13] K. Englehart, B. Hudgins, P. A. Parker, M. Stevenson, "Classification of the myoelectric signal using time-frequency based representations", Med. Eng. \& Phys., vol, 21, pp. 431-438, 1999.

[14] N. A. Arikidis, E. W. Abel and A. Forster, "Inter-Scale Wavelet Maximum - A Fine to Coarse Algorithm for Wavelet Analysis of the EMG Interference Pattern", IEEE Transactions on Biomedical Engineering, Vol. 49, pp 337-344. Apr. 2002.

[15] S. G. Mallat, "A wavelet tour of signal processing”, Academic Press, New York. 1998.

[16] S. Mallat and W. L. Hwang, "Singularity detection and processing with wavelets," IEEE Transactions on Information Theory, vol. 38, pp. 617-643, 1992.

[17] M. Unser, A. Aldroubi and M. Eden, "Fast B-spline transforms of continuous image representation and interpolation”, IEEE Trans. Pat. Anal. Mach. Intell., vol. 13, no. 3, pp. 227 285,1991

[18] M. Unser, A. Aldroubi and M. Eden, "On asymptotic convergence of B-spline wavelets to Gabor functions”, IEEE Trans. Info. Theory, vol. 38, no. 2, pp. 864-872, 1992.

[19] A. Aldroubi , M Unser., Wavelet in medicine and biology, CRC press, USA, 1996.

[20] HB. Mann, DR, Whitney, "On a Test of Whether One of Two Random Variables is Stochastically Larger than the Other". The Annals of Mathematical Statistics, vol 18,pp 50-60, 1947.

[21] E. Stalberg, J. Chu, V.Bril, S.D. Nandekar, S.Stalberg, M. Ericsson, "Automatic analysis of the EMG interference pattern”, Electroencephalograpy and Clinical Neurophysiology, vol 56, pp $672-681,1983$ 